Itinéraires Itinéraires

Littérature, textes, cultures

\title{
Éléments d'anthropo-logique : à propos des corpographies de Karl Lakolak
}

\section{Dominique Chevé}

\section{(2) OpenEdition}

1 Journals

Édition électronique

URL : http://journals.openedition.org/itineraires/398

DOI : 10.4000/itineraires.398

ISSN : 2427-920X

Éditeur

Pléiade

\section{Édition imprimée}

Date de publication : 1 mai 2009

Pagination : 181-189

ISBN : 978-2-296-08444-5

ISSN : $2100-1340$

\section{Référence électronique}

Dominique Chevé, «Éléments d'anthropo-logique : à propos des corpographies de Karl Lakolak», Itinéraires [En ligne], 2009-1 | 2009, mis en ligne le 13 juin 2014, consulté le 01 juillet 2020. URL : http:// journals.openedition.org/itineraires/398 ; DOI : https://doi.org/10.4000/itineraires.398

Ce document a été généré automatiquement le 1 juillet 2020

\section{cc)}

Itinéraires est mis à disposition selon les termes de la licence Creative Commons Attribution - Pas d'Utilisation Commerciale - Pas de Modification 4.0 International. 


\title{
Éléments d'anthropo-logique : à propos des corpographies de Karl Lakolak
}

\author{
Dominique Chevé
}

«... comme l'anthropologie, l'esthétique retrouve
la dialectique éternelle des échanges Vie-Mort
animés par le flux et le reflux de la matière
omniprésente dont on ne se libère pas »

(Thomas $1975: 158$ )

1 Provoqué, l'anthropologue ? Si nous nous accordons à dire, avec Jean-Luc Nancy, que « les corps sont des lieux d'existence » ou « donnent lieu à l'existence » mais également que la peinture "est l'art des corps parce qu'elle ne connaît que la peau...» (Nancy 2000: 16-17), que nous donnent à voir les œuvres de Karl Lakolak, " corpographe ", que mettent-elles en signes ? Et quels signes d'une anthropo-logique des corps ? Pour l'homme, Michel Leiris proposait "Somme de mots et de maux »1. Outre ceux-là, quels autres éléments offrent ces corps en images, cette profusion bigarrée ?

Qu'est-ce qu'un corps? Dirigeant une exposition récente ${ }^{2}$, Stéphane Breton s'interroge sur ce territoire où s'inscrivent toutes les écritures, sociales, symboliques et imaginaires. Question identique mais non générique pour des réalités différentes, essentiellement relationnelles, rétives ainsi à toute essentialisation comme support, chose, être ou « substance étendue " pour reprendre la formule cartésienne. Question par là même sans objet? Question inévitable pour autant que nous sachions la contextualiser, que nous la sachions métaphorique et toujours recommencée... territoire du vide ou du trop-plein. Le caractère kaléidoscopique, voire éclaté, de ce territoire de la corporéité ne peut échapper. Pour autant et pour chacun, le corps (le sien et celui de l'autre) demeure une énigmatique conjugaison d'évidences, parfois brutales, comme des perspectives infinies. Chacun de nos corps est construit selon une ontogénie individuelle, constitue un nœud d'ancrages et de contradictions, ordonne tous les possibles, de la douleur à la jouissance, de la maladie à la santé, de la 
monstruosité ou du handicap à la belle apparence et au plein usage de ses capacités comme à leur manque ou leur perte.

3 Tous les (nos) corps restent des corps poreux, plastiques et traversés en permanence : ni tout à fait dedans ou dehors, ni tout entièrement sujets, ni exclusivement objets mais surtout projets, ni individuels seulement, ni collectifs entièrement, hybrides. Faut-il en rester alors à une définition négative, une sorte d'« ontologie blanche » du corps ? Par nature, le corps serait rétif aux territoires fixes et ne répondrait alors qu'aux territorialisations fluides? Quel corps, du reste? Cette question persiste depuis les années 1970, moment où elle fut fixée par la désignation de la revue éponyme qui, déjà, s'interrogeait sur l'impossible objet-sujet de notre entendement, l'insoutenable tenue de sa réalité ontologique, sous la plume de Jean-Marie Brohm³ ${ }^{3}$ N'était-ce pas, en luimême alors, un fantôme infréquentable?

Où le rencontre-t-on, le corps ? Il ne peut plus être question de quête du corps humain, de rêver à une construction totalisante par l'anthropologie normative et européenne ou la philosophie $d u$ corps. L'évolution et la variabilité, l'histoire et la diversité, les constructions fictionnelles ou anatomiques et les expressivités socioculturelles, les corporéités et les matérialités, tout ce qui est du corps est frappé au coin de l'impossibilité de totaliser, d'unifier la chose. Au mieux, le corps est un «signifiant flottant " (Foucault 1978; 1986), résultant d'un bricolage, d'un feuilletage, même outrepassant ses limites physiques, dès lors qu'il est le nœud d'ancrage au monde et d'élaboration de ce monde pour chacun. Nous rencontrons des corps, pluriels et denses: corps désirés rendus désirables, corps fragiles et résistants, corps de brutes bardés de muscles et assignant leurs injonctions gueulardes à d'autres corps dominés, manipulés, affaiblis, corps mourants parce que vivants ${ }^{4}$. Nous rencontrons aussi des images de corps, non-corps spectacularisés, mais signifiés et rendus visibles pour une expérience sensible qui se prolonge dans une pratique et/ou une expérience esthétiques.

Confrontée aux Érographies d'incorporelles (Lakolak 2008) nous nous demandions ce qu'il restait des corps ainsi expeausés ${ }^{5}$ : rien, sinon justement l'infinie variation de la construction des corps déclinée dans cette sarabande singulière. Et c'est bien là ce qui interroge l'anthropologue renvoyé par cette œuvre à ses pauvres notions empêtrées et laides de «biosocioculturalité » des corps. Autre écriture, autre manigance, pour une autre saisie... le corps, lui, résiste et se bâtit de c(s)es résistances (Foucault $1986: 61-68$ ). C'est que cette tension permanente entre construction/dé-construction, recomposition, dévoilement et retrait, feuilleté des couches et éclat des couleurs, bref, au travers de ce kaléidoscope ( kaléidoscorps ${ }^{6} »$ ?) des corps bariolés, le corps semble se perdre, s'effacer à la surface de l'œuvre. Mais, outre que le singulier le désignant (« le » corps) est un leurre pour la rencontre désirée d'une chair singulière et ne peut satisfaire que la commodité d'un usage discursif, scientifique ou doxique, outre que la question «qu'est-ce qu'un corps?» demeure aporétique, ce qui résiste ce sont les corps, leur singulier pluriel, si l'on nous passe cet oxymore facile.

Des corps persistant dans leur opacité propre par-delà la surabondance de la violence chromatique; des corps maintenant leurs formes par-delà la fragmentation des collages, des découpes ; des corps se tenant bruts, nus, denses par-delà les maquillages, grimages et autres tatouages, par-delà les couches, traces, masques et reliefs ou brouillages et habillages de peinture et de tissu ou de plastique. Enfin, ces corps affirment leur(s) sexe(s) et leur(s) désir(s), leur(s) vie(s), par-delà l'hydridation du 
genre et de la mort, peut-être parce que, comme la mort, le désir donne forme, prend forme, est facteur de forme.

7 Quelque chose d'intact résiste. Dès lors, ce qui s'impose au regard devant ces mises en série du corps relève à la fois du vertige et de la reconnaissance. Vertige qui saisit nos imaginaires bridés au "rien de trop » dès lors qu'il s'agit de corps, alors qu'ils sont les lieux de tous les excès dionysiaques ou mortifères; reconnaissance d'une belle tentative insensée : faire exister les corps hybrides, accords et désaccords de nature et de culture, imposant comme une évidence cela même que notre culture ne peut réellement et véritablement (encore ?) penser et que nous vivons dans nos chairs, cette imbrication anthropo-logique. «Le corps est l'extension de l'âme jusqu'aux extrémités du monde et jusqu'aux confins du soi, l'un dans l'autre intriqués et indiscutablement distincts, étendue tendue à se rompre » écrit Jean-Luc Nancy (2003 : 28).

Dès lors, les corps de Karl Lakolak peuvent être textes, matières, formes, couleurs, éclats et fragments, crânes et visages, os et chairs, dedans et dehors : palimpsestes et série. Corps traversés d'échos tant historiques que symboliques: images christiques, masques mortuaires, planches anatomiques, héros guerriers, écorchés, arlequins étranges, victimes torturées, blessés de toutes les guerres, danseurs dionysiaques, corps tribaux... Corps répétés dont les déclinaisons attestent les invariants anthropologiques comme les variations : sexes, membres, postures, gestes, visages, squelettes, chairs... Au fond, il y a là matière à faire coexister diachronie et synchronie, jeux de l'altérité et du même. Ces images alors, pour le dire comme Giorgio Agamben (2004:32), chantent et récitent à la fois, viennent s'inscrire dans ce dépassement (non hégélien mais par la tension du métissage, la polyphonie des figures de l'art et des couleurs qui agissent, du bizarre qui transfigure ${ }^{7}$ ) que Warburg appelait de ses vœux en une " science sans nom " selon son diagnostic de schizophrénie tragique occidentale : la polarité représentée par la pratique magico-religieuse et celle de la contemplation mathématique, l'art et la science, ce qui ne signifie pas la rationalité s'opposant à l'irrationalité, bien évidemment ${ }^{8}$. Elles portent en elles mémoire des scènes primitives de l'art (et pas occidental seulement) comme images mnémoniques et sont toujours chargées d'une énergie capable d'(é)mouvoir, de troubler les corps acteurs (ceux des modèles, de l'artiste, des spectateurs). C'est tout un monde d'images qui est convoqué au travers de ces œuvres, jeux d'échos et de rappels, de transparences et de brouillages : survivances de la Vera Iconica (Marin 1993), des Madone, des crucifixions, de Marsyas, mais aussi des saints Sébastien blessés et rivés aux poteaux et aux colonnes, des statues de marbre de Palerme ou d'ailleurs, des Vanités, des figurines anthropomorphes aux Vénus des médecins, fermées/ouvertes du musée de La Specola, aux avatars enfin de toutes les incarnations, celle de Vishnou ou du Net, in effigie.

9 La perspective anthropologique peut donc ici emprunter le regard de Warburg sur les images, réalités dont l'inscription historique ne fait aucun doute mais qui appartiennent à la puissance symbolique de la pensée, font signes et sont productrices parce qu'elles traduisent des invariants de représentations dont il faut tenter de comprendre la nature et les sens, en fonction des contraintes symboliques à l'œuvre. Ces images ont alors une dimension transhistorique, voire une "a-chronicité » (DidiHuberman 1992 ; 2002). L'image est considérée comme trace des tensions spirituelles d'une culture, organe de la mémoire et de l'imaginaire collectifs'. Si, du point de vue de l'art, ce dévoilement du réel peut se passer de spéculations, du point de vue anthropologique, l'étude des œuvres ne vise pas leur "vérité ", mais les saisit comme 
archives sensibles exprimant les événements vécus, les peurs, les imaginaires et les croyances comme les interrogations, les confrontations et les savoirs des populations. L'approche anthropologique peut s'attacher devant ces images de Karl Lakolak aux traits structurels de la confrontation intersubjective et interhumaine, parce qu'elle impose de reconnaitre tout son sens à ce qui demeure, aux survivances, aux matrices invariables des images (Belting 2004) ${ }^{10}$.

Reste que la série de Karl Lakolak (2008) échappe à l'injonction anthropométrique de constitution de "l'homme moyen » chère à Quetelet et sauve, par l'art et l'atelier, de l'illusion abyssale de l'idéal type de Monsieur Tout-le-monde né dans les laboratoires. Mais elle échappe aussi aux Anthmpométries de Klein qui tente de faire que le corps fixe lui-même son image, dans la fusion ou confusion des « femmes-pinceaux ». Karl Lakolak met l'homme au pied du vide en des sortes de Vanités corporelles : confrontation des regards, des face-à-face, des moments, des expériences. Le crâne, du reste, objet privilégié tant des Vanités que des anthropologues, jamais anodin qu'il soit adamique ou archive biologique des fouilles ou des charniers, est prégnant dans certaines de ces œuvres, en flagrant délit de ricanement.

11 Ce qui surprend encore renvoie aux entrelacs de tous les rituels corporels (mais y a-t-il des rituels qui ne le soient pas ?) ceux des «transes chamaniques ou films de sciencefiction (Lafargue, 2008 : 17) comme au métissage des forces, des formes et des matières. Sorte de parade infinie, ces œuvres mettent en scène et en signes la geste d'une humanité archaïque et postmoderne à la fois : danse corporelle, comme l'on dit «danse macabre ", et qui rythme et égrène les pluriels des états, des postures, des statuts et des sens humains. L'ecce homo en singularités multipliées des corps. C'est pourquoi ces œuvres provoquent le regard, l'interrogation de l'anthropologue pour lequel il ne fait aucun doute depuis longtemps que le corps est la visée et le creuset de toutes les prescriptions et proscriptions sociales et culturelles, des pratiques rituelles, symboliques et thérapeutiques, ancrage des logiques du sensible, de la socialité comme des relations sémiotiques avec le monde.

12 Tout se passe comme si ces corps concentraient et éclataient à la fois les pratiques humaines fondamentales: sexualité, écriture/parole, parure et ritualisation, soin, connaissance, séduction, jeu, travestissement, etc. Or, ce qui se lie en elles et par ces corps, ce sont ordre et désordre. Pourrions-nous alors ici faire un parallèle entre le bouffon rituel, le sacrificateur et l'artiste? Les trois opèrent des transformations : l'un transmue le désordre en ordre, l'autre fait de la vie avec de la mort, de l'ordre avec de la violence, le dernier enfin mêle les forces en des incarnations fugitives dont les images demeurent. Marie-Anne Paveau écrit à propos des érographies de Karl Lakolak : "Un art fait au corps, qui incorpore le langage, lui donne corps et un corps.» (Lakolak $2008: 23$ )

13 Nous retrouvons alors cet invariant de toutes les sociétés, procédé de liaison de l'ordre et du désordre dans lequel les anthropologues reconnaissent une anthropo-logique. Toutes les pratiques d'inversion sociale qui, par un contre-cérémonial, renversent les puissants pour les rétablir à nouveau dans l'ordre qu'ils dominent et dans leurs privilèges, qui font des fous les héros des lieux sacrés, de la chair renversée un renforcement du pouvoir institué, qui renouvellent l'ordre établi par la provocation subversive ${ }^{11}$.

14 Sexe, Sacré et Fortune, voilà une trilogie qui structure les humains et leurs sociétés, selon Georges Balandier (2003). Voilà un triptyque constitutif, nous semble-t-il, de 
l'œuvre de Karl Lakolak. Une version colorée, exultante et polyphonique, dionysiaque de l'affirmation joyeuse d'une grande santé d'Éros. Des beaux corps dénudés, une sensualité lisible, des styles de vie et d'être corporel sinon revendiqués, du moins affirmés.

Cette "collection" de corps, et le terme "collection" renvoie sans nul doute à la puissance dominatrice et obstinée de l'artiste dans le creuset de son atelier, ordonne une manifestation sacrée. Ce «mélange de crainte et d'attachement, cette attitude ambiguë que détermine l'approche d'une chose à la fois attirante et dangereuse, prestigieuse et rejetée, cette mixture de désir et de terreur » par quoi Michel Leiris (1979: 60) définissait ce qui peut passer pour le signe psychologique du sacré saisit ici. Ces signes du sacré tiennent tant aux visages exposés qu'aux corps investis par l'artiste et offerts au regard. La singularité du visage convoque la distinction individuelle, l'altérité et d'emblée, le sens (Lévinas 1967; Simmel 1988: 140 ; Le Breton 1992). Chez Lévinas, le visage signifie par lui-même, sans médiation ni renvoi au contexte, il est « expérience pure, expérience sans concept » (Lévinas 1967 : 177). Le visage est à la fois une présence et un horizon d'attente. Ici, il incarne à la fois le mystère et la transparence, dès lors que l'artiste le rend lisible par la surabondance de traitements divers (maquillages, grimages, écritures, masques et autres casques transparents de film plastique, bandelettes, tatouages, etc.) et qu'il impose le face à face.

Enfin, nous savons, depuis l'étude devenue incontournable de Mary Douglas (1971), que l'ordre symbolique associe sacré et souillure, pureté et impureté. Le corps est, par excellence, le lieu de ces associations, sang, sperme, lait, larmes et autres humeurs s'y répondent et s'y confondent ou s'y excluent, dans des systèmes de représentations complexes. Les liquidités comme les traces dégoulinantes ou la peau maculée renvoient à ces humeurs corporelles, toujours investies symboliquement dans les cultures, jamais laissées au hasard, comme la sexualité. Elles sont ce par quoi une humanité advient en s'arrachant à la nature et en fondant une culture, mais portent témoignage des évidences biologiques, physiologiques toujours signifiantes.

Cependant, ces corps travaillés par l'artiste nous sont offerts en images. À proprement parler, il ne s'agit ni de performances, ni d'installations, mais d'images de peintre/ photographe. Du reste, les corps sont voués par avance au régime de l'image et à l'écriture rapsodique de la chair. Il nous semble alors que se rejoue dans les représentations de Karl Lakolak le paradoxe de la dualité irréductible et originaire de l'image comme du corps, de l'image corporelle et du corps imagé. Énigme de notre culture, celle de l'union du sensible et de l'intelligible, du multiple et de l'un, qui se noue tant dans l'image que dans le corps, par l'un et l'autre ${ }^{12}$. Dans l'aspect sériel des œuvres de Karl Lakolak, les images comme les corps sont répétables et reproductibles mais, en même temps, sont apparitions d'un unique. "Toute image repose sur un abîme... » écrit Giorgio Agamben (2004 : 139), cet abîme justement qui existe entre le répétable et l'unique ; tout corps également. Tout corps est surdéterminé, saturé de sens et de signes, traversé par des forces sociales et identitaires, éclaboussé de couleurs et de griffures, voilé et dévoilant les strates de ses affects, vibrant de ses intensités, chargé de tous les mots et maux de la tribu. L'artiste le prend pour le mettre à l'épreuve alors que le modèle et le spectateur lâchent prise pour mieux se (dé)livrer. 


\section{BIBLIOGRAPHIE}

Agamben, G., 2004, Image et mémoire. Écrits sur l'image, la danse et le cinéma, Paris, Desclée de Brouwer.

Balandier, G., 2003, Civilisés, dit-on, Paris, PUF.

Belting, H., 2004, Pour une anthropologie des images, Paris, Gallimard.

Brohm, J.-M., 1986 [Présentation], Quel corps ?, Paris, Éditions de la Passion, p. 7-15.

Claudot Hawad, H. et Lafargue, B., 2007, Introduction, Corps et couleurs, $\mathrm{n}^{\circ} 3$ de CORPS, Revue interdisciplinaire, Paris, Dilecta.

Didi-Huberman, G., 1999, Ouvrir Vénus. Nudité, rêve, cruauté, Paris, Gallimard.

-, 2002, L'Image survivante : histoire de l'art et temps des fantômes selon Aby Warburg, Paris, Minuit, coll. « Paradoxe».

-, 2007, L'Image ouverte. Motifs de l'incarnation dans les arts visuels, Paris, Gallimard, coll. « Le temps des images ».

Douglas, M., 1971, De la souillure, Paris, Maspero.

Foucault, M., 1984 [1976], Histoire de la sexualité, Paris, Gallimard, t. 1, La Volonté de savoir, 1976, t. 2, t. 3 .

-, 1986, « Pouvoir et corps », Quel corps ?, Paris, Les Éditions de la Passion, p. 61-68.

Gombrich, E. H., 1997, Aby Warburg: An Intellectual Biography, Londres, Phaidon Press.

Jankélévitch, V., 1977, La Mort, Paris, Flammarion.

Lafargue, B., 2008, « Dépeindre les mille plateaux d'un corps » dans Lakolak, 2008, p. 15-18.

Lakolak, K., 2007, Sarabande pour un corps, Alfortville, Everland.

-, 2008. Érographies d'incorporelles, Alfortville, Everland.

Le Breton, D., 1992, Des visages. Essai d'anthropologie, Paris, Métailié.

Leiris, M., 1979, « Le sacré dans la vie quotidienne », dans D. Hollier, Le Collège de sociologie, Paris, Gallimard.

-, 1985, Langage, tangage ou ce que les mots me disent, Paris, Gallimard.

Lévinas, E., 1967, En découvrant l'existence avec Husserl et Heidegger, Paris, Vrin (2éd.).

Marin, L., 1993, Des pouvoirs de l'image. Gloses, Paris, Le Seuil.

-, 1994. De la représentation, Paris, Le Seuil.

Nancy, J.-L., 2000, Corpus, Paris, Métailié, coll. « Sciences Humaines ».

-, 2003. «L'extension de l'âme » dans Descartes, Carnets le Portique, p. 9-28.

Simmel, G., 1988, « La signification esthétique du visage » dans La Tragédie de la culture, Paris, Rivages.

Thomas, L.-V., 1975, Anthropologie de la mort, Paris, Payot. 


\section{NOTES}

1. Emprunt au glossaire de Michel Leiris, Langage, tangage (1985).

2. 2006, musée du Quai Branly.

3. Sur le rabat de première de couverture, le responsable éditorial se demande déjà : «De quoi parle-t-on quand il est question du corps ? » et en fait le projet de recherche de la revue et de son élaboration théorique (voir Brohm 1986).

4. L'oxymore qui a nourri toute la poésie baroque et a existé sous la plume de Montaigne pour lequel on ne meurt pas de ce qu'on est malade mais bien de ce qu'on est vivant, est ici repris de Vladimir Jankélévitch, qui écrivait du corps : « ... la source des maladies et le lieu de la souffrance et le principe de la finitude et de l'usure temporelle. À la lettre, le vivant meurt de son corps » (Jankélévitch 1977 : 450).

5. Le néologisme est utilisé par Bernard Lafargue (Érographies d'incorporelles, Karl Lakolak, Alfortville, Everland, 2008, p. 17 et, à notre connaissance, crée par Jean-Luc Nancy dans Corpus (op. cit.). L'auteur écrit que «le corps est l'être-exposé de l'être» (p.32) et prise au moins en un sens premier, cette expression nous paraît pouvoir lui être empruntée sans trop en trahir la conception philosophique.

6. Selon l'expression de ce néologisme par H. Claudot Hawad et Bernard Lafargue, dans leur introduction de « Corps et Couleurs » (2007).

7. Voir l'étude de Bernard Lafargue dans Lakolak 2007.

8. Voir pour ses belles analyses des textes de Warburg, Giorgio Agamben, notamment Image et Mémoire (2004) et particulièrement son texte : "Aby Warburg et la science sans nom ». Pour la connaissance des inédits de Warburg, voir la «biographie intellectuelle» publiée par E. H. Gombrich (1997) ou encore l'ouvrage majeur de Didi-Huberman, L'Image survivante: histoire de l'art et temps des fantômes selon Aby Warburg, 2002.

9. Dans son étude sur Warburg, Giorgio Agamben remarque que « le symbole et l'image ont selon Warburg la même fonction que, chez Semon, celle de l'engramme dans le système nerveux central de l'individu : en eux se cristallisent une charge énergétique et une expérience émotive qui surviennent comme un héritage transmis par la mémoire sociale et qui, pareilles à l'électricité condensée dans une bouteille de Leyde, deviennent effectives au contact de la volonté sélective d'une époque déterminée » (2004: 18-19). C'est l'image, plus que l'œuvre d'art qui est saisie par Warburg pour ce qu'il appelle un «diagnostic de l'homme occidental ». À ce propos, G. Didi-Huberman fait un état exhaustif des travaux de Warburg (voir DidiHuberman 2002).

10. Nous ne saurions développer davantage ce point, plus propre à l'anthropologie des images, ce que nous avons fait ailleurs, pour d'autres horizons, plus «scientifiques ». Reste une lecture, fondamentale dans cette approche également, celle de l'ouvrage de Hans Belting, Pour une anthropologie des images, 2004.

11. Les travaux des anthropologues à cet égard sont très abondants. Un choix fondé serait ici impossible. Retenons, par dette personnelle, les travaux de Georges Balandier (2003).

12. Voir à ce sujet les très belles analyses de Louis Marin, 1993/1994. Sur cette question également, la littérature est très abondante, citons par exemple les ouvrages de G. DidiHuberman, notamment Ouvrir Vénus et L'Image ouverte (1999 et 2007). 


\section{RÉSUMÉS}

Confrontés aux Érographies d'incorporelles de Karl Lakolak, nous nous demandons ce qui reste des corps ainsi exposés et sur la peau desquels il écrit : rien, sauf précisément l'infinie variation sur la construction même des corps, dans une sarabande singulière. Les anthropologues ne peuvent que se tourner vers leur pauvre petite notion, confuse et laide, de «bio-socio-culturalité » des corps. À travers ce kaléidoscope de corps variés, le corps lui-même semble disparaître, s'estomper à la surface de l'œuvre. Cependant, les corps se maintiennent dans leur opacité particulière, au-delà de l'excès de la violence chromatique: des corps qui gardent leur forme au-delà de la fragmentation des collages et des découpages; des corps, mal dégrossis, nus, denses derrière leur maquillage, leurs déguisements et autres marquages, au-delà des couches, des traces, des masques et des reliefs ou du brouillage et du costume de peinture, de tissu ou de plastique. En bref, ces corps affirment leur(s) sexe(s) et leur(s) désir(s), leur vie, au-delà de l'hybridation des genres et de la mort, peut-être parce que, comme la mort, le désir donne forme, prend forme, est facteur de forme. L'œuvre de Lakolak positionne l'homme au bord des abysses dans Vanités Corporelles qui se réfère à l'entrelacement de tous les rituels aussi bien qu'au métissage des forces, des formes, des humeurs et des matériaux : Ecce homo dans les singularités multiples du corps.

Faced with Karl Lakolak's Érographies d'incorporelles, we ask what is left of the bodies thus exposed and on whose skins he writes: nothing, except precisely the infinite variation on the very construction of bodies, in a singular saraband. Anthropologists can only turn to their poor little notions, muddled and ugly, of "bio-socio-culturality" of bodies. Through this kaleidoscope of variegated bodies, the body itself seems to vanish, to fade away at the work's surface. Yet, bodies continue in their peculiar opaqueness, beyond the excess of chromatic violence: bodies that maintain their shapes beyond the fragmentation of collages and cut-ups; bodies, rough-hewn, naked, dense beyond their make-ups, disguises, and other markings, beyond the layers, the traces, the masks and the reliefs or the scrambling and costuming-paint, fabric, or plastic. In short, these bodies assert their sexe(s) and their desire(s), their lives, beyond gender hybridization and death, perhaps because, like death, desire gives shape, takes shape, is a factor of shape. Lakolak's work puts man at the edge of the abyss in Corporeal Vanities that refer to the intertwining of all rituals as well as to the miscegenation of forces, shapes, humors, and materials: Ecce homo in the bodies' multiple singularities.

\section{INDEX}

Mots-clés : anthropo-logique, corps, bioculturalité, métissage, collection, rituel

Keywords : anthropo-logic, body, bioculturality, miscegenation, collection, ritual

\section{AUTEUR}

\section{DOMINIQUE CHEVÉ}

UMR 6578 CNRS, Université de la Méditerranée, GDR 2322 CNRS 